\title{
Der Effekt des Molekularen Prägens auf die Porengrößenverteilung von Polymeren
}

Cornelia Hettrich ${ }^{1}$, Franziska Grüneberger ${ }^{1}$, Soeren Schumacher ${ }^{1}$, Carola Fanter ${ }^{2}$, Nenad GajovicEichelmann $^{1}$

${ }^{1}$ Fraunhofer Institut für Biomedizinische Technik, 14476 Potsdam, Am Mühlenberg 13

${ }^{2}$ Fraunhofer Institut für Angewandte Polymerforschung, 14476 Potsdam, Geiselbergstr. 69

\section{Zusammenfassung}

Poröse Polymere sind zu einem wichtigen Meilenstein bei der Entwicklung fortschrittlicher Materialien mit erweiterter Funktionalität erwachsen. Anwendungen dieser Materialien reichen über chemische und biologische Separationsmedien und Membranen zu Vorlagen für die Nanomaterialsynthese und in die Sensortechnologie.

Durch das molekulare Prägen werden hochporöse Polymere (MIPs) erzeugt, die in der Lage sind, die erkennungsspezifischen Strukturen ihrer Targetmoleküle in den Hohlräumen zu konservieren. Hierbei wird das Targetmolekül zum Templat, welches einen Komplex mit polymerisierbaren Monomeren eingeht. Dieser Templat-Monomer-Komplex wird dann in Gegenwart eines hohen Anteil Vernetzers und einem porogenen Lösungsmittel polymerisiert, um so eine starre Hülle mit dem Abdruck des Templates zu formen. Die Entfernung des Templates führt zu einem molekular geprägten Polymer, welches wie ein Rezeptor das Templat selektiv binden kann.

Molekular geprägter Polymere mit Fruktose und Pinakol als Template werden mit einem polymerisierbaren Benzoboroxolderivat (MABX) als Funktionsmonomer und mit TRIM als Vernetzer im klassischem BulkVerfahren zur Erkennung von Fruktose im physiologischen $\mathrm{pH}-$ Bereich hergestellt. Die Wiederanbindung von Fruktose wird bei pH 7,4 (0.1 M Phosphatpuffer, 10\% MeOH) getestet.

Die ermittelten Daten werden mit denen eines Kontrollpolymers (CP) verglichen, welches nur den Vernetzer TRIM enthält.

Der Einfluss der Porengrößenverteilung von Polymeren mit und ohne Prägung auf die Erkennungsleistung der MIPs wird untersucht.

\section{Einleitung}

Die Morphologie der MIPs wird maßgeblich durch das porenbildende Lösungsmittel bestimmt. Art und Volumen des Lösungsmittels, das hier auch als Porogen bezeichnet wird, sind variabel. Häufig verwendete Lösungsmittel bei der MIP-Synthese sind: THF, Acetonitril, Dioxan und Gemische aus Acetonitril und Toluol.

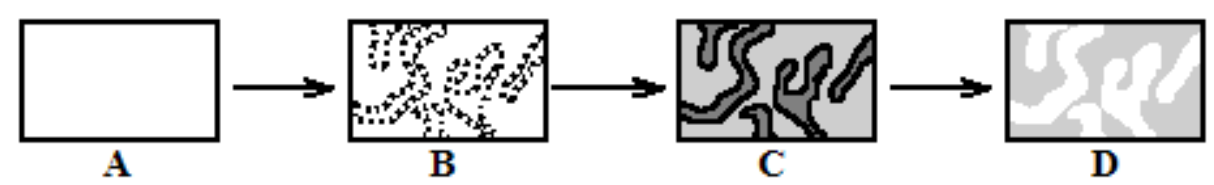

Bild 1 Funktionsweise eines porogenen Lösungsmittels

Die Aufgabe des Porogens ist das anfängliche Lösen aller Monomere (A, Abb. 1). Während der Polymerisation (B) findet Schritt für Schritt eine Phasentrennung (C) statt, weil sich das bildende Polymer im Gegensatz zum Monomer nicht im Porogen löst. Nach der Polymerisation wird das Lösungsmittel durch Abdampfen entfernt und es bleibt eine poröse Struktur (D) im Polymer zurück. ${ }^{[1]}$ Durch Variation der 
Zusammensetzung der Reaktionsmischung hat sich herausgestellt, dass ein 1:1-Verhältnis (w/v) zwischen Monomeren und Porogen gut geeignet ist, um spezifische und gut zugängliche Hohlräume zu bilden. ${ }^{[2]}$ Bei diesem hohen Lösungsmittelanteil werden besonders viele sogenannte Meso- und Makroporen (2 - $100 \mathrm{~nm})$ gebildet, wodurch die Gesamtoberfläche der Polymere mit 50 bis $1000 \mathrm{~m}^{2} / \mathrm{g}$ ausgesprochen groß ist.

\section{Methoden und Material}

\subsection{Synthese der Polymere}

Für die Synthese der geprägten Polymere werden zuerst der entsprechende Boronsäureester ( 2 mmol) und AIBN (1.8 mmol) in einem $20 \mathrm{~mL}-S e p t e n g l a s$ in $9 \mathrm{~mL}$ Dioxan suspendiert. Nach der Zugabe von TRIM $(26.5 \mathrm{mmol}, 9 \mathrm{~g}, 8.5 \mathrm{~mL})$ wird die Mischung $10 \mathrm{~min}$ mit Argon durchspült. Anschließend wird die Polymerisation bei $65^{\circ} \mathrm{C}$ im Ultraschallbad gestartet, danach $48 \mathrm{~h}$ bei dieser Temperatur im Trockenschrank belassen und weitere $24 \mathrm{~h}$ bei $95^{\circ} \mathrm{C}$ gehalten.

Tafel 1 Darstellung der MIPs

\begin{tabular}{|c|c|c|}
\hline \multirow{2}{*}{ Funktionsmonomer } & Templat & MIP \\
\cline { 2 - 3 } & Fruktose & MABX(Fru)-MIP \\
MABX(Pin)-MIP
\end{tabular}

Die Kontrollpolymere CP, welche nur den Vernetzer enthalten, werden analog zur MIP-Synthese, aber in verschiedenen Lösungsmitteln bzw. Gemischen: Acetonitril/Toluol 1:1, Dioxan und THF, hergestellt.

Der Polymermonolith wird nach der Polymerisation mechanisch zerkleinert und anschließend feucht mit Aceton in die Korngrößenfraktionen $<25 \mu \mathrm{m}, 25-50 \mu \mathrm{m}$, und $>50 \mu \mathrm{m}$ klassiert.

Das Herauswaschen des Templates aus der gewünschten Fraktion $25-50 \mu \mathrm{m}$ erfolgt durch Spülen mit Wasser/Methanol 1:1 (3x40 mL) bzw. Wasser (3x40 mL), darauffolgender Zentrifugation (10 min, 3500 x g) und Dekantieren. Das Polymer wird im Exsikkator unter Vakuum getrocknet.

\subsection{Oberflächen- und Porengrößenbestimmung}

Die Bestimmung der spezifischen Oberfläche der Polymere erfolgt durch Adsorption und Desorption von Stickstoff an einem Sorptomat 1900 der Fa. Fisons Instruments.

\section{Ergebnisse}

\subsection{Fruktosebindung an die MIPs}

Die Bindungseigenschaften für Fruktose bei $\mathrm{pH} 7.4$ an das MABX(Fru)-MIP und an das MABX(Pin)-MIP werden in einem Batch-Binding Experiment untersucht. Als Negativkontrolle wird das Kontrollpolymer CP5 verwendet. Dazu wird eine definierte Menge Polymer mit einer definierten Menge Fruktose in $0.1 \mathrm{M}$ Phosphatpuffer versetzt, das Reaktionsgefäß über Nacht schüttelnd stehen gelassen und zentrifugiert. Der Überstand wird mit Szintillationsflüssigkeit versetzt. Auf diese Weise kann die in der Lösung ungebundene Fruktose bestimmt werden, da die eingesetzten Fruktoselösungen zuvor mit ${ }^{3} \mathrm{H}$-Fruktose dotiert worden ist. ${ }^{[3]}$ Unter Kenntnis der eingesetzten Fruktosekonzentration und der ermittelten freien Fruktose kann die gebundene Fruktose berechnet werden.

Am Kontrollpolymer CP5 werden keine unspezifischen Wechselwirkungen der Fruktose mit dem Trägermaterial nachgewiesen.

Das Bild 2 zeigt die Konzentrationsabhängigkeit der Bindung von Fruktose an das MABX(Fru)-MIP und das MABX(Pin)-MIP.

Fruktose bindet 2,5-mal stärker an das Fruktose-geprägte Polymer als an das MABX(Pin)-MIP. 


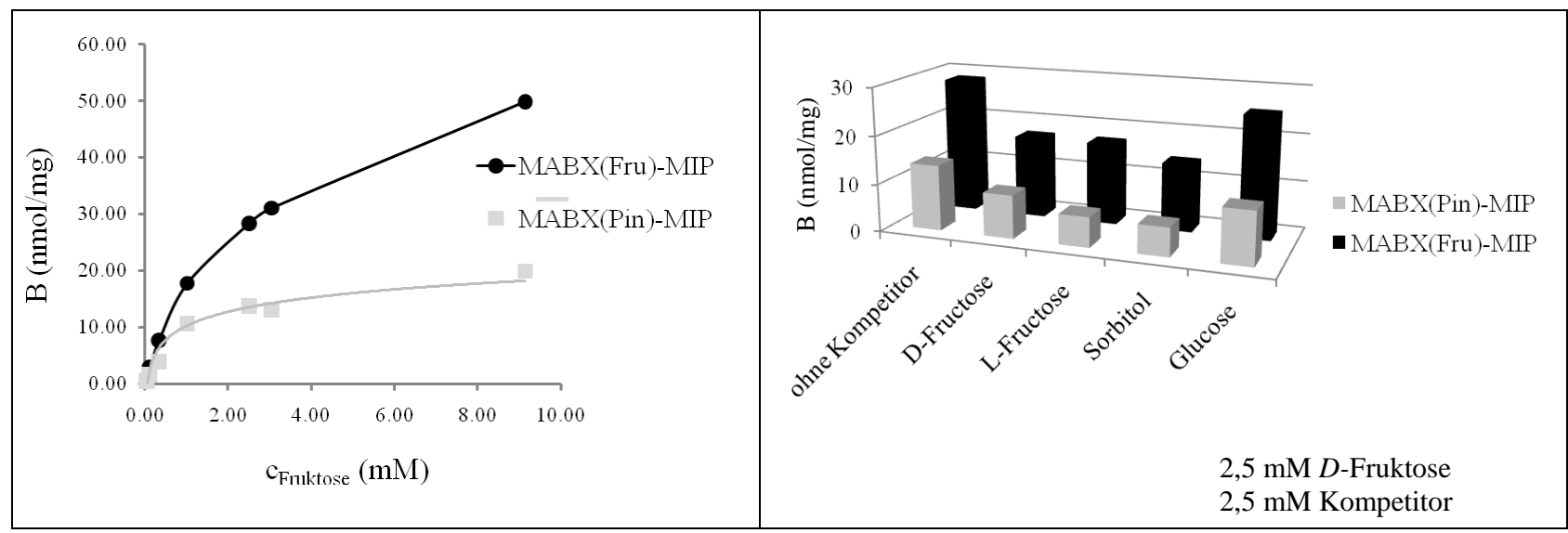

Bild 2 Vergleich der D-Fruktoseanbindung an die MIPs bei $\mathrm{pH} 7,4$

Bild 3 D-Fruktoseanbindung in Anwesenheit konkurrierender Saccharide und Sorbitol

Da bei beiden Polymertypen $2 \mathrm{~mm}$ Boronsäureester als Templat eingesetzt werden, sollte auch die Zahl der gebildeten Bindungstaschen identisch sein. Doch die Zahl der Haftgruppen pro Tasche ist unterschiedlich. Die Pinakol-MIPs tragen nur eine Boronsäure und die Fruktose-MIPs zwei Boronsäuren pro Bindungsstelle. Im MABX(Pin)-MIP kann die Fruktose demnach nur eine Einpunktbindung eingehen und wird daher nicht so spezifisch gebunden.

In der Tafel 2 sind die erreichten Bindungskapazitäten der synthetisierten MIPs den theoretisch möglichen Bindungsstellen gegenübergestellt, welche anhand der bekannten Zusammensetzung des Polymers berechnet werden konnten. Für diese Berechnung wird angenommen, dass zum einen alle Bindungsstellen gut zugänglich sind und zum anderen alle als Templat dienenden Fruktosemoleküle erfolgreich herausgewaschen werden konnten.

Tafel 2 Wiederbelegungsraten der einzelnen Polymere bei $\mathrm{pH}$ 7,4

\begin{tabular}{ccc}
\hline MIP & MABX(Fru)-MIP & MABX(Pin)-MIP \\
\hline $\begin{array}{c}\text { theoretisch besetzbare } \\
\text { Bindungsstellen } B_{\max }\end{array}$ & $195 \mathrm{nmol} / \mathrm{mg}$ & $208 \mathrm{nmol} / \mathrm{mg}$ \\
\hline $\begin{array}{c}\text { Wiederbelegungsraten im } \\
\text { Verhältnis zu B }\end{array}$ & $26 \%$ & $10 \%$ \\
\hline
\end{tabular}

\subsection{Oberflächen-und Porengrößenbestimmung der MIPs}

Die spezifischen Oberflächen nach der BET-Methode und die Volumina der Meso- $(2-50 \mathrm{~nm})$ bzw. Mikroporen $(<2 \mathrm{~nm})$ nach der BJH- bzw. HK-Methode sind in Tafel 3 dargestellt.

Tafel 3 Ergebnisse der $\mathrm{N}_{2}$-Sorptionsmessungen der Kontrollpolymere und der MIPs

\begin{tabular}{lccc}
\hline \multicolumn{1}{c}{$\begin{array}{c}\text { Kontrollpolymere }(\mathrm{CP}) \\
\text { MIP-Polymere }\end{array}$} & $\begin{array}{c}\text { BET-Oberfläche } \\
{\left[\mathrm{m}^{2} / \mathrm{g}\right]}\end{array}$ & $\begin{array}{c}\text { BJH Mesoporen- } \\
\text { volumen }[\mathrm{mL} / \mathrm{g}]\end{array}$ & $\begin{array}{c}\text { HK-Mikroporen- } \\
\text { volumen [mL/g] }\end{array}$ \\
\hline CP1 (THF- 9mL) & 498 & 0.322 & 0.237 \\
CP2 (Acetonitril/Toluol 1:1 - 9mL) & 482 & 0.422 & 0.227 \\
CP3 (Acetonitril/Toluol 1:1 - 5mL) & 297 & 0.099 & 0.145 \\
CP4 (THF, Ultraschall - 9mL) & 724 & 0.489 & 0.351 \\
CP5 (Dioxan, Ultraschall - 9mL) & 646 & 0.409 & 0.297 \\
MABX(Fru)-MIP (THF - 9mL) & 436 & 0.213 & 0.207 \\
MABX(Fru)-MIP (Dioxan, Ultraschall - 9mL) & 457 & 0.210 & 0.279 \\
MABX(Pin)-MIP (Dioxan, Ultraschall - 9mL) & 520 & 0.247 & 0.238 \\
\hline
\end{tabular}


Die Kontrollpolymere CP1 und CP2, welche wie die geprägten Polymere mit $9 \mathrm{~mL}$ Lösungsmittel auf $8.5 \mathrm{~mL}$ TRIM hergestellt wurden, zeigen eine ähnliche spezifische Oberfläche mit Werten von 498 bzw. $482 \mathrm{~m} / \mathrm{g}$ im gleichen Größenbereich. Die Angaben für die kumulativen Porenvolumina für die Meso- als auch Mikroporen sind ebenfalls annähernd gleich groß. Somit sollte ein ähnlich guter Stofftransport zu den Bindungsstellen im Polymer gewährleistet sein. Die Art des Porogens ist hier weniger signifikant.

Das CP3 mit $5 \mathrm{~mL}$ Lösungsmittel je $8.5 \mathrm{~g}$ TRIM zeigt eine mittelmäßige Stickstoffadsorption und hat mit $297 \mathrm{~m}^{2} / \mathrm{g}$ eine um $2 / 5$ minimierte spezifische Oberfläche als CP1 und CP2. Bei den kumulativen Porenvolumina ist der Unterschied noch gravierender. Hier zeigt das Porogen deutlich seinen Einfluss.

Die Synthese der beiden Kontrollpolymere CP4 und CP5 wurde im Unterschied zu den oben genannten im Ultraschallbad durchgeführt. Diese neue Methode führte zu einer verbesserten Durchmischung der Monomere in der Polymerlösung und schließlich zu einem hochporösen Polymer.

Die Gegenüberstellung der geprägten Polymere zeigt, dass deren spezifische Oberflächen unter denen der Kontrollpolymere CP1 und CP5 liegen, das mit den zusätzlich in der Reaktionsmischung vorhandenen Funktionsmonomeren bei konstant gebliebenem Lösungsmittelvolumen zu erklären ist. Das mit Pinakol als Templat geprägte Polymer hat mit $520 \mathrm{~m}^{2} / \mathrm{g}$ die größte spezifische Oberfläche. Das MABX(Fru)-MIP hat bei vergleichbaren Mesoporenvolumen einen höheren Anteil an Mikroporen, welches in guter Korrelation mit dessen Bindungsverhalten zu Fruktose ist.

\subsection{Kreuzreaktivität des MABX(Fru)-MIP mit anderen Sacchariden}

Bild 3 stellt die Spezifität der Bindung von Fruktose an das MABX(Fru)-MIP bzw. deren Kreuzreaktivität gegenüber anderen Sachariden ( $L$-Fruktose, $D$-Glukose) dar. Zusätzlich wurde Sorbitol wegen seiner hohen Affinität zu Boronsäuren ausgewählt.

Da nur die $D$-Fruktose in radioaktiv markierter Form zur Verfügung stand, wurde ein sogenanntes Kompetitionsexperiment durchgeführt. In diesem Fall wurde $D$-Fruktose zusammen mit einem der anderen Saccharide zum Polymer gegeben und untersucht, wie viel Fruktose, welche mit dem anderen Saccharid um die Bindungsstelle konkurriert, gebunden wird.

Da Sorbitol die höchste Bindungsaffinität zu Boronsäuren hat, hindert es die Fruktose bei der Wiederanbindung am meisten, gefolgt von $L$-Fruktose. Die Fruktose-Anbindung findet in Gegenwart von $D$ Glukose fast unbeeinträchtigt statt.

Es wurde eine klare Korrelation der Bindungskapazität mit dem kumulativen Mikroporenvolumina und der spezifischen Oberfläche gefunden. MABX(Fru)-MIP hat die höchste Menge an Mikroporen, die eine hohe Polymernetzwerkstruktur bedingen und damit die gute Erreichbarkeit der Bindungsstellen unterstützen.

Die Art des Lösungsmittels hat einen Einfluss auf die Morphologie, aber nicht auf die Selektivität der geprägten Polymere. Die Affinität der Haftgruppe, hier des Benzoboroxols bestimmt die Bindungseigenschaften der MIPs entscheidend.

\section{Danksagung}

Die Nachwuchsgruppe „Biomimetrische Materialien und Systeme“ dankt dem BMBF für die finanzielle Unterstützung (BMBF Grant 0311993).

\section{Literatur}

[1] D.C. Sherrington: Chem. Commun. 21, 2275-2286, 1998

[2] G. Wulff; Angew. Chem. 107, 1958-1979, 1995

[3] F. Grüneberger, Diplomarbeit 2010, Universität Potsdam 